\title{
Experimentelle Studien über den Einfluss der nervösen Innervation auf den Stoff= und Gasaustausch durch die Kapillaren.
}

II. Mitteilung. Versuch an Uran- und Kantharidinhunden.

\author{
Von \\ T'sunao Watanabe. \\ (渡部網男)
}

(Aus der Medizinischen Klinik von Prof. Dr. T. Kato, Tōhoku Reichsuniversität zu Sendai.)

In der ersten Mitteilung ${ }^{1)}$ konnte im Stauungsversuch am Hund bereits der Nachweis erbracht werden, dass nach Nervendurchschneidung schon in einigen Stunden(3Stunden) oder zu einerZeit(3 Wochen), wo am Gewebe degenerative Veränderungen auftreten, im dem betreffenden Nerven zugehörigen Kapillargebiet erhebliche Permeabilitätssteigerung sich geltend macht und ferner im Stoff- und Gasaustausch zwischen Blut und Gewebe komplizierte Veränderungen sich einstellen.

Nach gegenwärtigen Anschauungen unterliegt es mehr keinem Zweifel, dass Uran und Kantharidin, sog. Nierengifte auch Gewebsgifte im weiteren Sinne darstellen und deshalb die Gewebszellen überhaupt sowie auch die Kapillaren schädigen. Heinecke u. Meyerstein ${ }^{2)}$, Pollak ${ }^{3}$ und auch Naito ${ }^{4)}$, Yamaguchich und Kimura ${ }^{6}$ ) an hiesiger Klinik haben bei Vergiftungen mit eben genannten Giften in der Richtung des intermediären Stoffaustausches und Gaswechsels zu Einflüssen der Giftwirkung Stellung genommen. Die Ergebnisse von obigen Forschern lassen sich kurz dahin zusammenfassen, dass beim Uran die Kapillarschädigung überwiegt, während dem Kantharidin

1) Wat a n a be, Tohoku Journ. Exp. Med., 1938, 32, 77.

2) Heinecke a. Meyerstein, Dtsch. Arch.f. klin. Med., 1907, 90, 18.

3) Pollak, Arch. f. exp. Pathol. u. Pharm., 1923, 97, 352.

4) Naito, Tohoku Journ. Exp. Med., 1924-1925, 5, 351.

5) Y a magu chi, Tohoku Journ. Exp. Med., 1927, 9, 73.

6) K i mu r a, Tohoku Journ. Exp. Med., 1930, 15, 199. 
freilich neben dieser kapillarschädigenden Wirkung zugleich auch die Eigenschaft, die Gewebszellen stark zu lädieren, deutlicher zukommt, es scheint jedenfalls zwischen der Wirkung der beiden Gifte ein gewisser Unterschied zu bestehen.

In vorliegender Versuchsreihe wurde unter Anwendung der mit Uran oder Kantharidin genügend vergifteten Hunde der Stauungsversuch am Schenkel der einen Seite unter ein und demselben Verfahren wie es in der I. Mitteilung angewandt wurde, ausgeführt. Es wurden dann nach denselben Methoden, wie im vorausgegangenen Versuch am venösen Schenkelblut die Hämoglobin- und Eiweisskonzentration, Serumkochsalz, der kolloid-osmotische Druck (unten kurz als k. o. D. bezeichnet), der $\mathrm{O}_{2}$-Gehalt sowie der Blutstrom in der Femoralvene bestimmt. Nachdem man dadurch das Verhalten der Permeabilität der betreffenden Kapillaren sowie die Verhältnisse des intermediären Stoffund Gasaustausches analysiert hatte, wurde einem jeden Hund der N. ischiadicus auf der anderen Seite durchschnitten und 3 Stunden danach der gleiche Stauungsversuch an diesem entnervten Schenkel angestellt und entsprechende Daten ermittelt, um ein Bild über Veränderungen im intermediären Stoffaustausch zwischen Blut und Gewebe durch den Ausfall nervöser Innervation zu verschaffen. In anderer Versuchsreihe wurde analoger Stauungsversuch nach dreiwöchigem Ablauf nach der Denervation, wo an zugehörigen Geweben und Kapillaren degenerativatrophische Veränderungen ausgesprochen auftraten, ausgeführt.

Die Technik der Versuchsmethode wurde schon in der ersten Mitteilung ausführlich geschildert, weshalb die Beschreibung darüber hier unterlassen werden kann.

\section{Versuch an Uranhund.}

Als Versuchsmaterial wurden 10 gut ernährte Hunde verwendet. Sie wurden in 2 Gruppen eingeteilt, die eine Gruppe wurde zum Versuch nach dreistündigem Ablauf nach der Ischiadicusdurchschneidung gebraucht, während eine andere Gruppe zum Versuch nach dreiwöchigen Ablauf benutzt wurde. Die Uranvergiftung wurde in der Weise herbeigeführt, dass einem jeden Versuchstier eine 5\%ige Uranylnitratlösung im Mengenverhältnis von $0,05 \mathrm{ccm}$ pro $\mathrm{kg}$ Körpergewicht subkutan injiziert wurde. Nachdem man bei der ersten Gruppe von Hunden 30 Stunden nach der Uraninjektion den Stauungsversuch am Unterschenkel einer Seite ausgeführt und das Versuchstier im Käfig ungefähr 18 Stunden lang (also ca. 48 Stunden nach Uranvergiftung) be- 
lassen hatte, hat man den Ischiadicus der anderen Seite durchschnitten und nach dreistündigem Ablauf den Stauungsversuch am Schenkel der betreffenden Seite erneut vorgenommen.

Bei der zweiten Gruppe von 5 Hunden, denen vor 3 Wochen der $\mathrm{N}$. ischiadicus auf einer Seite durchschnitten worden war, wurde etwa 50 Stunden nach der Uranvergiftung der Stauungsversuch am durch Denervation atrophierten Schenkel durchgeführt.

\section{Staungsversuch an Uranhund vor der Ischiadicusdurchschneidung.}

In vorliegender Versuchsreihe wurde an 5 Uranhunden zuerst darüber untersucht, ob und inwieweit Hämoglobin, Serumeiweiss, k. o.D. und Serumkochsalz sowie $\mathrm{O}_{2}$-Unsättigung des venösen Blutes durch Uranvergiftung von entsprechenden Werten, die in der ersten Mitteilung an gesunden Hunden gewonnen wurden, abweichende Veränderungen aufweisen (Tab. 1 und I. Mitteilung, Tab. 1). Übersichtshalber sind hier erhaltene Durchschnittswerte in Tab. 2 zusammenfassend dargestellt.

Hämoglobin wurde durch Uranvergiftung im Durchschnitt um 13,3\% vermehrt gefunden. Versuch 1 stellt einen einzigen Ausnahmefall dar, in welchem Hämoglobin nach dreiwöchigem Ablauf nach der Nervendurchschneidung ausserordentlich abnahm. Diese Abnahme dürfte durch die Anämie, die während der Beobachtungsfrist aus irgendwelchen unbekannten Ursachen zustande gekommen wäre, bedingt sein. Wenn man also von diesem Ausnahmefall absehe, so würde sich die prozentuale Zunahme des Hämoglobins, welche nach dreiwöchigem Ablauf nach der Nervendurchschneidung auftrat, noch viel höher beziffern als in der Tab. 1 aufgezeichnete Zifferwerte. Aus Feststellungen von $\mathrm{Kylin},{ }^{7)} \mathrm{Naito^{4 }}$ und $\mathrm{Yamaguchi},{ }^{5)}$ die durch Uranund Kantharidinvergiftung eine gesteigerte Permeabilität der Kapillaren nachgewiesen haben, geht hervor, dass eine derartige Bluteindikkung dadurch verursacht wird, dass das durch Uranwirkung geschädigte Gewebe aufquillt und Wasser aus dem Blut durch die abnorm permeabel gewordenen Kapillaren hindurch an sich reisst.

Serumeiweiss nahm zu, die prozentige Zunahme betrug im Mittel $15,7 \%$, lag also nahe an derselben des Hämoglobins. Druck pro \% erniedrigte sich deshalb, weil der k.o.D. trotz erheblicher Zunahme des

7) Kylin, Zbl. f. inn. Med., 1920, 41, 505: 1922, 43, 41. 


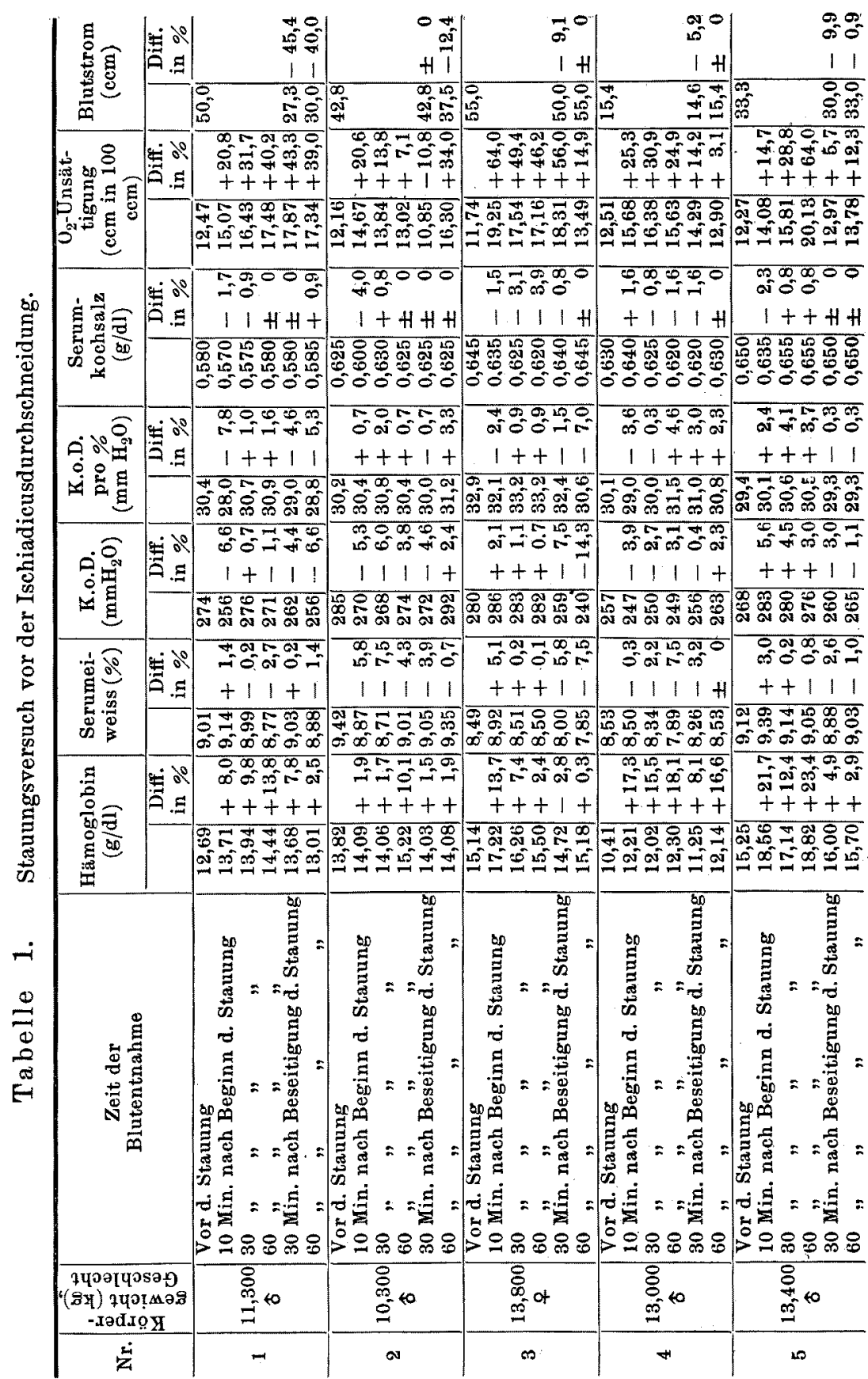


Eiweisses fast keinen Schwankungen unterworfen war. Diese beträchtliche Erniedrigung des Durcks pro \% dürfte darauf bezogen sein, dass unter anderem durch den extravasalen Austritt von kleinmolekulären Eiweissteilchen infolge der durch Uran bewirkten Steigerung der Kapillarpermeabilität grossmolekuläre Eiweissteilchen in Blut zunahm. Utber die auffallende Erniedrigung des Drucks pro \%, die bei Uranvergiftung auftritt, wurde schon von Kimura u. Nakazawa ${ }^{8}$ ) sowie Saito $\mathrm{u}$. Nakazawa ${ }^{9}$ und auch von $\mathrm{Yasuda^{10) }}$ nachgewiesen und veröffentlicht, weshalb ich hier darauf nicht eingehen mag.

Serumkochsalz wurde auch vermehrt gefunden, was mit dem Ergebnis von $\mathrm{Kim}$ ura $^{6}$ ) übereinstimmt.

Der Blutstrom und die $\mathrm{O}_{2}$-Unsättigung venösen Blutes bleiben bei der Uranvergiftung fast unbeeinflusst.

Der demnächst ausgeführte Sta u ung s vers u ch ergab folgendes (Tab. 1).

Hämoglobin : Hämoglobin nahm durch Stauung zu. Die maximale Zunahme betrug 10,1-23,4\%, war also offenbar grösser als die beim gesunden Hund ermittelte Zunahme (2,1-15,9\%). Die Hämoglobìnkonzentration nahm durch Stauung in 2 von 5 Versuchen (Versuch 1 und 2) allmählich zu, das Maximum wurde in einem Versuch (Versuch 3) nach 10 Minuten, in übrigen 2 Versuchen (Versuch 4 und 5) nach 30 Minuten erreicht. Sofort nach der Beseitigung der Stauung fiel die Hämoglobinkonzentration ohne Ausnahme ab, und zwar ging sie in einem Versuch (Versuch 4) nach vorausgegangenem Abfall wieder in die Höhe.

Serumseiweiss: Die Veränderung des Serumeiweisses verhielt sich ausserordentlich verschieden von derselben beim gesunden Hund. Serumeiweiss erfuhr nämlich, im Gegensatz zum Hämoglobin, welches in allen Fällen gleichermassen zunahm, durch Stauung eher eine Abnahme. In einigen Fällen stieg das Serumeiweiss am Beginn der Stauung allerdings transitorisch mehr oder weniger an, um dann jedoch nach 30 oder 60 Minuten wieder abzusinken. Diese Beziehung ist aus dem Vergleich mit den in Tab. 3 in der I. Mitteilung aufgezeichneten Daten ersichtlich. K.o.D. war in 3 von 5 Versuchen (Versuch 1, 2 und 4) erniedrigt, in 2 Versuchen (Versuch 3 und 5) erhöht. Von diesen 2 Fällen zeigte ein Fall (Versuch 3) eine äusserst geringe Erhöhung, so dass man sagen kann, dass der k.o.D. nur in einem einzi-

8) Kimura u. Nakazawa, Tohoku Journ. Exp. Med., 1930, 16, 352.

9) Saito u. Nakaza wa, Tohoku Journ. Exp. Med., 1932, 19, 252.

10) Yasuda, Tohoku Journ. Exp. Med., 1937, 31, 456. 
Tabelle

Durchschnittswerte vor und

\begin{tabular}{|c|c|c|c|c|c|c|c|c|c|}
\hline \multirow[b]{2}{*}{$\therefore$} & \multicolumn{3}{|c|}{ Hämoglobin (g/dl) } & \multicolumn{3}{|c|}{ Serumeiweiss $(\%)$} & \multicolumn{3}{|c|}{ K.o.D. $\left(\mathrm{mmH}_{2} \mathrm{O}\right)$} \\
\hline & 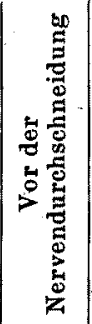 & 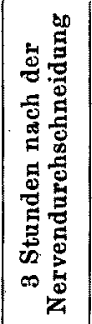 & 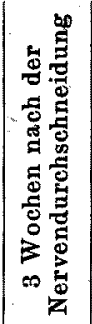 & 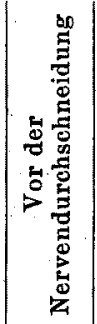 & 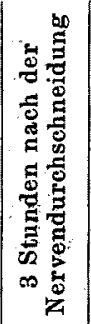 & 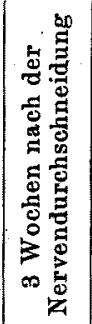 & 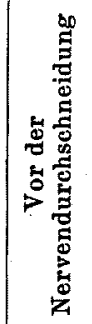 & 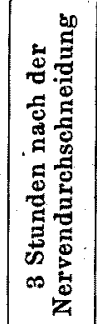 & 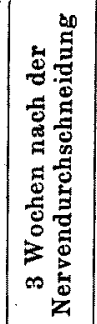 \\
\hline & $\begin{array}{c}\operatorname{Max} . \\
15,25 \\
\operatorname{Min} . \\
10,41\end{array}$ & $\begin{array}{c}\text { Max. } \\
17,36 \\
\text { Min. } \\
11,00\end{array}$ & $\begin{array}{c}\text { Max. } \\
24,36 \\
\text { Min. } \\
8,32\end{array}$ & $\begin{array}{c}\text { Max. } \\
9,42 \\
\text { Min. } \\
8,49\end{array}$ & $\begin{array}{c}\text { Max. } \\
\mathbf{9}, 42 \\
\text { Min. } \\
7,02\end{array}$ & $\begin{array}{c}\text { Max. } \\
10,10 \\
\text { Min. } \\
7,05\end{array}$ & $\begin{array}{c}\text { Max. } \\
285 \\
\text { Min. } \\
257\end{array}$ & $\begin{array}{c}\text { Max. } \\
285 \\
\text { Min. } \\
190\end{array}$ & $\mid \begin{array}{c}\text { Max. } \\
324 \\
\text { Min. } \\
209\end{array}$ \\
\hline Durehschnitt & 13,46 & 13,85 & 17,64 & 8,91 & 8,55 & 8,41 & 273 & 243 & 238 \\
\hline Diff. in $\%$ & & $+2,9$ & $+31,0$ & & $-4,0$ & $-5,6$ & & $-11,0$ & $-12,8$ \\
\hline Norm & 11,88 & 12,57 & 13,28 & 7,70 & 8,35 & 8,41 & 256 & 313 & 295 \\
\hline $\left.\begin{array}{c}\text { Proz. Zu- u. Abnahme } \\
\text { gegenüber d. Norm }\end{array}\right\}$ & $+13,3$ & $+10,2$ & $+32,8$ & $+15,7$ & $-2,4$ & \pm 0 & $+6,6$ & $-22,4$ & $-19,3$ \\
\hline
\end{tabular}

gen Fall (Versuch 5) erhöht war, d.h. der k.o.D. durch Stauung mit Ausnahme eines einzigen Falls herabgesetzt ist. Druck pro \% erhöhte sich gewissermassen; es war aber keine gesetzmässige Beziehung herauszufinden, immerhin steht soviel fest, dass es keinen Fall gab, der eine so deutliche Erföhung wie in der Norm aufwies.

Serumkochsalz zeigte gegenüber dem normalen Wert fast keine Verschiedenheit.

$\mathrm{O}_{2}$-Defizit, also die $\mathrm{O}_{2}$-Unsättigung venösen Blutes erfuhr durch Stauung eine Vermehrung, die im Vergleich zum Normalwert einigermassen stärker war. Während die $\mathrm{O}_{2}$-Unsättigung des venösen Blutes in der Norm durch Beseitigung der Stauung eher mehr als vor der Stauung abnahm, kam beim Uranhund, ausgenommen einen Fall (Versuch 2), solche Abnahme niemạls vor. Blutstrom beim Normalhund stieg nach Behebung der Stauung über den Vorwert hinaus, wohingegen er beim Uranhund hierbei im allgemeinen unter den Vorwert abfiel und erst dann an denselben heranrïckte.

Von grossem Interesse ist die Tatsache, dass die Hämoglobinkonzentration durch Stauung recht erheblich zunahm, während die Ei- 
2.

nach der Nervendurchschneidung.

\begin{tabular}{|c|c|c|c|c|c|c|c|c|c|c|c|}
\hline \multicolumn{3}{|c|}{ K.o.D. pro $\%\left(\mathrm{mmH}_{2} \mathbf{0}\right)$} & \multicolumn{3}{|c|}{ Serumkoohsalk $(g / d l)$} & \multicolumn{3}{|c|}{$\begin{array}{l}\mathrm{O}_{\mathrm{g}} \text {-Unsättigung } \\
(\mathrm{ccm} \text { in } 100 \mathrm{ccm})\end{array}$} & \multicolumn{3}{|c|}{ Blutstrom (ccm) } \\
\hline 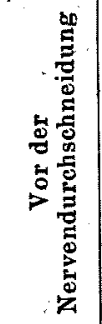 & 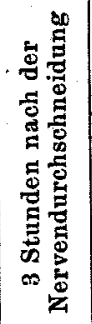 & 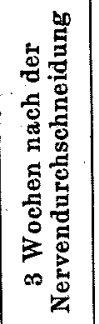 & 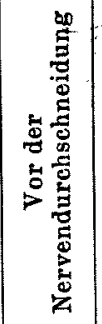 & 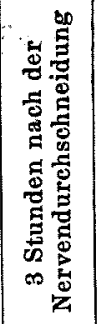 & 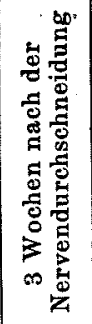 & 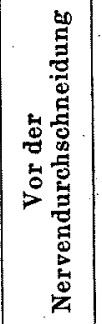 & 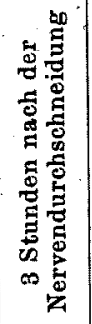 & 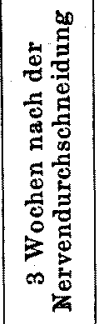 & 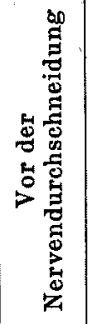 & 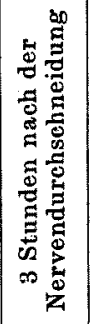 & 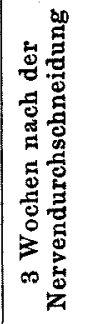 \\
\hline $\begin{array}{r}\text { Max. } \\
\mathbf{3 2 , 9}\end{array}$ & $\begin{array}{r}\operatorname{Max} . \\
31,0\end{array}$ & $\begin{array}{r}\operatorname{Max} . \\
\mathbf{3 2 , 1}\end{array}$ & $\begin{array}{c}\text { Max. } \\
0,650\end{array}$ & $\begin{array}{c}\operatorname{Max} . \\
0,670\end{array}$ & $\begin{array}{c}\text { Max. } \\
0,720\end{array}$ & $\begin{array}{c}\text { Max. } \\
12,51\end{array}$ & $\begin{array}{c}\operatorname{Max} . \\
13,22\end{array}$ & $\begin{array}{r}\operatorname{Max} . \\
17,62\end{array}$ & $\begin{array}{r}\operatorname{Max} . \\
55,0\end{array}$ & \begin{tabular}{|}
$\operatorname{Max}$. \\
50,0
\end{tabular} & $\underset{33,6}{\operatorname{Max} .}$ \\
\hline$\underset{29,4}{\operatorname{Min} .}$ & $\underset{26,2}{\operatorname{Min} .}$ & \begin{tabular}{|} 
Min. \\
$\mathbf{2 5 , 1}$
\end{tabular} & $\underset{0,580}{\text { Min. }}$ & $\mid \begin{array}{c}\text { Min. } \\
0,625\end{array}$ & $\begin{array}{l}\text { Min. } \\
0,580\end{array}$ & $\begin{array}{c}\text { Min. } \\
11,74\end{array}$ & Min. & $\begin{array}{c}\text { Min. } \\
11,78\end{array}$ & $\begin{array}{c}\text { Min. } \\
\mathbf{3 3}, 3\end{array}$ & Min. & $\underset{6,1}{\text { Min. }}$ \\
\hline 30,6 & 28,4 & 28,2 & 0,626 & 0,644 & 0,661 & 12,23 & 12,73 & 14,97 & & & \\
\hline 33,5 & $-7,2$ & $-7,8$ & 0,568 & $\begin{array}{r}+6,1 \\
0,593\end{array}$ & $+5,6$ & 11,16 & $\begin{array}{r}+\quad 4,1 \\
6,05\end{array}$ & $\begin{array}{r}+22,4 \\
10,60\end{array}$ & $\left\{\begin{array}{c}\text { Max. } \\
60,0 \\
\text { Min. }\end{array}\right.$ & $\begin{array}{c}\text { Max. } \\
75,0 \\
\text { Min. }\end{array}$ & $\begin{array}{l}\text { Max. } \\
50,0 \\
\text { Min. }\end{array}$ \\
\hline$-8,6$ & $-23,9$ & $-19,9$ & $+9,3$ & $+12,0$ & $+13,4$ & $+9,6$ & $+110,4$ & $+41,2$ & 20,0 & 0,0 & 34,6 \\
\hline
\end{tabular}

weisskonzentration hingegen dadurch im allgemeinen abnahm. Die Zunahme der Hämoglobinkonzentration deutet auf den Übertritt von grossem Quantum Blutwassers ins Gewebe hin, die Abnahme der Eiweisskonzentration spricht dafür, dass das Bluteiweiss zusammen mit dem Blutwasser ins Gewebe abfiltriert worden ist. Es ist dies nämlich ein Beweis dafür, dass durch Uranvergiftung die Permeabilität der Kapillaren für Wasser und Kolloide enorm gesteigert ist. Für oben erwähnte Zunahme der Hämoglobinkonzentration dürfte meines Erachtens der Umstand, dass das Gewebe, welches unter dem Einfluss der Uranvergiftung steht, unter Bedingungen von Stauung, also mangelhafter $\mathrm{O}_{2}$-Zufuhr und $\mathrm{CO}_{2}$-Anhäufung in stärkerem Masse als das gesunde Gewebe quillt und Wasser aus dem Blut gierig anzieht, auch einen der Faktoren abgeben. Die Erscheinung, dass der Eiweissgehalt trotz bestehender intensiver Bluteindickung durch Stauung abnimmt, deutet offenbar darauf hin, dass der Austritt des Eiweisses mit ausgesprochener Leichtigkeit vor sich geht, d.h. die Kapillarendothelien durch Wirkung des Urans kolloiddurchlässig geworden ist. 
2. Staungsversuch nach dreistüdigem Ablauf nach der Ischiadicusdurchschneidung.

Den oben angeführten 5 Uranhunde wurde 15-18 Stunden nach Beendigung des vorherigen Versuchs (also etwa 50 Stunden nach Uranvergiftung)der N.ischiadicus auf der anderen Seite durchschnitten und 3 Stunden später am venösen Femoralblut der betreffenden Seite einschlägige Grössen bestimmt. Daran anschliessend wurde der Stauungsversuch an ein und demselben Schenkel ausgeführt.

Aus Rücksicht darauf, dass zwischen dem vorherigen und dem diesmaligen Versuch ein Unterschied in der Zeitdauer der Vergiftung besteht, wurden zur Beurteilung der vorliegenden Versuchsergebnisse nicht allein Werte vor und nach der Ischiadicusdurchschneidung miteinander verglichen, sondern zugleich auch mit Werten, die in der I. Mitteilung an normalen Hunden nach der Ischiadicusdurchschneidung gewonnen wurden, vergleichend betrachtet.

Um daraufhin zu prüfen, welche Veränderungen das in der zugehörigen Unterextremität kreisende Blut beim Uranhund, der der Ischiadicusdurchschneidung unterworfen worden ist, aufweisen würde, hat man hier erhaltene Werte mit Werten, die an ein und demselben Uranhund vor der Ischiadicusdurchschneidung gefunden wurden, verglichen. Dieser Vergleich ergab, dass Hämoglobin, Serumeiweiss, Serumkochsalz und $\mathrm{O}_{2}$-Unsättigung des venösen Blutes sowie der Blutstrom gegenüber den Werten vor diesem Eingriff nicht so sehr abweichende Veränderungen aufwiesen, während der k.o.D. und Druck pro $\%$ deutlich abnahmen. Während beim gesunden Hund der k.o.D. und Druck pro \% durch Nervendurchschneidung erhöht waren, waren entsprechende Grössen beim Uranhund dadurch mehr oder weniger erniedrigt. Dies dürfte dadurch bedingt sein, dass kleinmolekuläre Eiweissteilchen durch Ichiadicusdurchschneidung einigermassen mehr ins Gewebe übergetreten ist.

Beim Normalhund trat durch Nervendurchschneidung am venösen Blut neben der Steigerung des Blutstromes beträchtliche Abnahme der $\mathrm{O}_{2}$-Unsättigung auf; dieser Befund aber klang nach einigen Wochen allmählich ab (siehe unten). Allein beim Uranhund wurden selbst gleich nach der Nervendurchschneidung bezüglich des Blutstromes und der $\mathrm{O}_{2}$-Unsättigung des venösen Blutes nennenswerte Veränderungen vermisst. Bei der Uranwirkung resultiert aller Wahrscheinlichkeit nach die Gefässchädigung derart intensiv, dass es durch die Nervendurchschneidung nicht zur Gefässerweiterung kommt, wie 
dies bei der Norm der Fall ist, dementsprechend dürften auch an dem Blutstrom und der $\mathrm{O}_{2}$-Unsättigung des venösen Blutes, wie oben geschildert, Veränderungen nicht so ausgeprägt zum Ausdruck kommen, wie beim Normalen.

Staungsversuch: Es wurde dann an den oben erwähnten der Ischiadicusdurchschneidung unterworfenen Hunden Staungsversuch ausgeführt (Tab. 3). Hämoglobin nahm durch Stauung erheblich zu; die Zunahme belief sich auf 14,3-28,7\%, war also gegenüber dern Wert (3,6-16,8\%), der am gesunden Hund unter denselben Bedingungen ermittelt wurde, weitgehend grösser. Nach Behebung der Stauung fiel die Hämoglobinkonzentration ab, nicht aber unter den Anfangswert, um dann wieder meistens den Weg zur Erhöhung einzuschlagen.

Als am normalen Hund mit durchschnittenem N. ichiadicus der Stauungsversuch angestellt wurde, erfuhr das Serumeiweiss nicht allein während des Stauungsversuchs, sondern auch nach Beseitigung desselben stets Zunahme. Beim Uranhund hingegen stieg das Serumeiweiss zwar während derersten 10-30 Minuten nach Beginn der Stauung an, aber am Ende des Versuchs, nämlich nach 60 Minuten ab; besonders in 4 von 5 Versuchen (Versuch 1, 3, 4 und 5) trat eine mehr als vor der Stauung deutliche Eiweissverdünnung in Erscheinung. Ein derartiges Verhalten des Eiweisses trat niemals parallel mit demselben des Hämoglobins auf. Die Eiweisszunahme, die am Ende des Stauungsversuchs auftrat, bestand in der Mehrzahl der Fälle nach Beseitigung der Stauung, weiter fort.

Die Veränderung des k.o.D. richtete sich im grossen und ganzen nach derselben des Eiweisses. K.o.D. erhöhte sich nämlich schon 10 30 Minuten nach Beginn des Staungsversuchs und auch nach 60 Minuten, um dann im Verein mit der Eiweissabnahme abzusinken. Weil die Erhöhung des k.o.D. indessen höhergradig als die des Eiweisses war, stieg Druck pro \% während der Stauung an, fiel aber gegen Ende des Staungsversuchs allmählich ab, um nach Abschluss desselben in der Regel um ein Geringes unter den Ursprungswert abzusinken.

Die Veränderung des Serumkochsalzes verhielt sich nicht so abweichend von derselben beim Normaltier.

Die $\mathrm{O}_{2}$-Unsättigung venösen Blutes wuchs durch Stauung zu, dieser Zuwachs war aber gegenüber dem Normaltier geringer. Sie trat nach Beseitigung der Stauung graduell an das fruhere Niveau heran. Das Verhalten des Blutsroms nach Beseitigung der Stauung richtete 


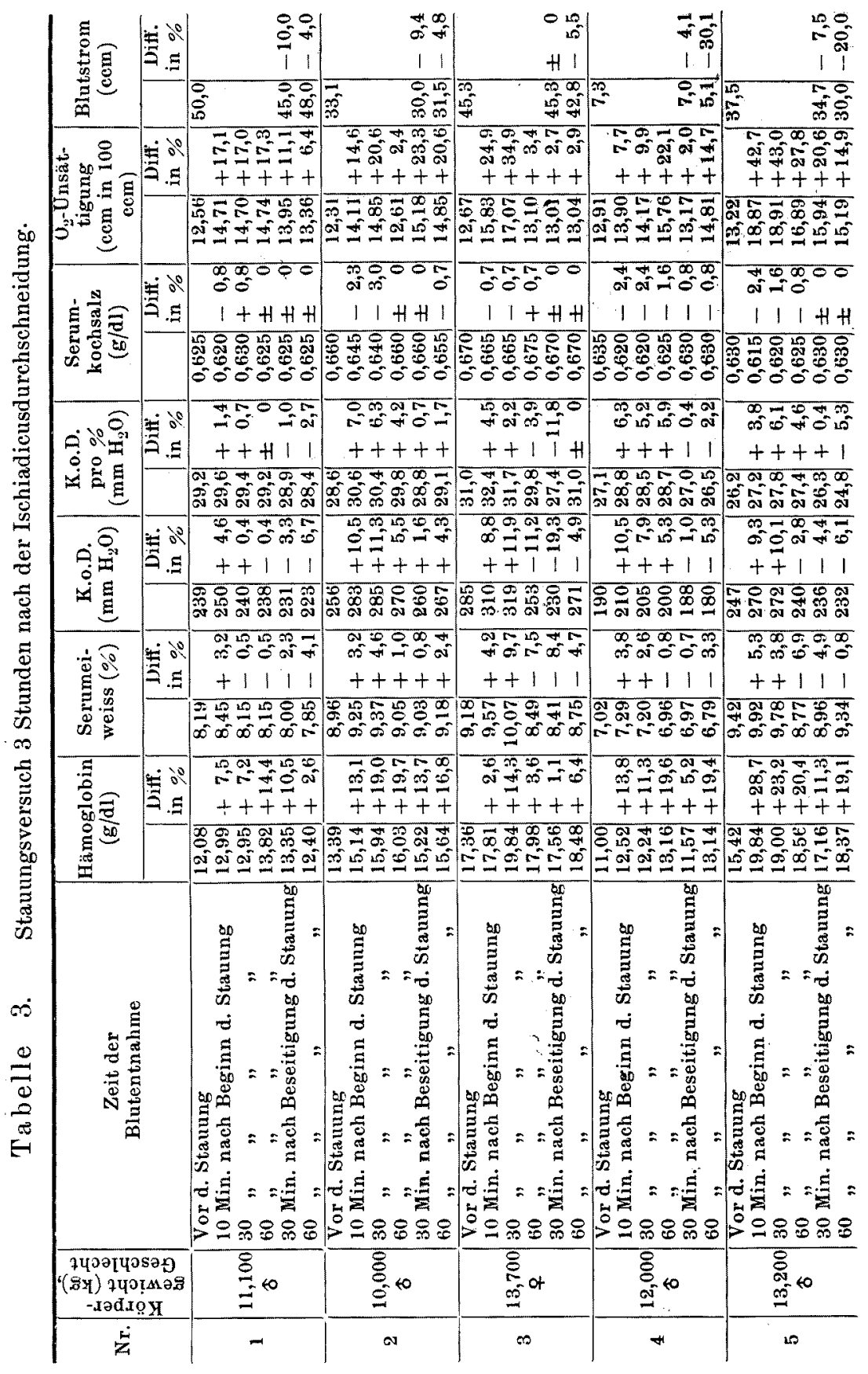


sich beinahe ähnlich demselben beim Urantier mit intaktem N. ischiadicus.

Oben geschilderte Befunde seien nun im folgenden unter Vergleich mit Werten, die an normalen Hunden mit durchschnittenem Ischiadicus im Stauungsversuch gewonnen wurden, und ausserdem auch mit Daten, die an Uranhund mit intaktem Ischiadicus im einschlägigen Versuch ermittelt wurden, einer theoretischen Betrachtung unterzogen.

Aus erheblicher Erhöhung der Hämoglobinkonzentration ist wohl darauf zu schliessen, dass beim Uranhund mit durchschnittenem Ischiadicus die Permeabillität des zugehörigen Kapillargebietes für Wasser enorm gesteigert ist. Die durch den Einfluss der Stauung hervorgebrachte stärkere Gewebsquellung dürfte wahrscheinlich an oben erwähnter Zunahme der Hämoglobinkonzentration beteiligt sein.

Was die Veränderungen des Eiweisses anbelangt, ist die Zunahme des Serumeiweisses transitorischer Natur und zudem im allgemeinen recht geringfügig, während das Hämoglobin durch Stauung sich sehr erheblich und dauernd vermehrt. Schon von Behebung der Stauung beginnt-das Eiweiss abzunehmen und fällt unter dem Vorwert herab. Dies deutet darauf hin, dass bei der Stauung das Bluteiweiss mit Leichtigkeit und in vermehrten Mengen durch die Kapillarwand hindurch ins Gewebe abfiltriert wird. Die Differenz zwischen dem Zunahmeverhältnis des Hämoglobins und dem des Eiweisses stellt einen Gradmesser für die Permeabilität der Kapillarwand für Eiweiss dar. Diese Permeabilitätssteigerung ist stärker sowohl als bei der Stauung an normalem Hund mit durchschnittenem Ischiadicus wie auch als bei der Stauung am Uranhund mit intaktem Ischiadicus. Die am Anfang der Stauung auftretende geringfügige Eiweisszunahme ist dadurch bedingt, dass die Kapillardurchlässigkeit in dieser Phase noch nicht genügend gesteigert ist, so dass Wasser allein durch die Kapillarwand frei durchtreten kann, während der Durchtritt des Eiweisses aber mehr oder weniger behindert wird. Die nach Behebung der Stauung noch fortbestehende Abnahme des Eiweisses ist wohl darauf zurückzuführen, dass die durch Uran geschädigten Kapillarendothelien durch Stauung mechanisch aufgelockert und ausserdem durch Hinzutreten des Sauerstoffmangels an ihrer Permeabilität noch mehr gesteigert sind und sich nach Beseitigung der Stauung von diesem abnormen Zustand nicht leicht erholen könmen. Da diese gesteigerte Durchlässigkeit für Kolloid vor der Beseitigung der Stauung sehr ausgesprochen aufgetreten ist, so stellt sich gegen Ende des Stauungsversuchs der inten- 
sive extravasale Austritt des Eiweisses ein und führt ofters zum $\mathrm{Ab}$ fall des Bluteiweissgehaltes unter den Wert vor der Stauing.

Im übrigen der Umstand, dass der k.o.D. einigermassen stäker als die Zunahme des Eiweisses erhöht ist, und dementsprechend auch Druck pro \% in der Mehrzahl der Fälle in die Höhe geht, weist darauf hin, dass hierbei Moleknlaraggregate des Bluteiweisses nach kleiner Seite hin verschoben sind.

\section{Staungsversuch nach dreiwöchigem Ablauf nach der Ischiadicusdurchschneidung.}

In vorliegender Versuchsreihe wurde das Versuchstier 3 Wochen nach Ischiadicusdurchschneidung mit Uran vergiftet und etwa 50 Stunden später der Stauungsversuch an der Extremität mit durchschnittenem Ischiadicus ausgeführt.

An 5 Hunden gewonnene Versuchsergebnisse sind in Tab. 4 angegeben.

Man vergleiche zuerst in Tab. 2 die Daten bevor der Stauungsversuch durchgeführt wird, mit denen beim Normalhund. Hierbei ersieht man, dass beim Uranhund die Erhöhung der Hämoglobinkonzentration und die Zunahme des Serumkochsalzes besonders ausgeprägt zutage treten, während das Serumeiwess fast keine grosse Veränderung aufweist und der k.o.D. und Druck pro \% sich deutlich erniedrigen, ferner dass die $\mathrm{O}_{2}$-Unsättigung mehr oder weniger zunimmit. Wenn man weiterhin diese Daten mit den kurz nach der Ischiadicusdurchschneidung erhaltenen Daten vergleicht, so ergibt sich, dass nach dreiwöchigem Ablauf das Hämoglobin stärker zunimmt, während der k.o.D. und Druck pro \% in noch erheblịcherem Masse absinken. Der Blutstrom verringert sich gegenüber dem Wert beim Normalhund gewissermassen.

Wenn man jetzt die Ergebnisse im Stauungsversuch kurz zusammenfasst (Tab. 4), so nimmt das Hämoglobin durch Stauung zu ; diese Zunahme von 4,8-18,5\% ist gegenüber dem Wert (9,2-22,0\%), der beim gesurden, unter denselben Bedingungen befindlichen Hund gefunden wird, gewissermassen kleiner und weiterhin auch im Vergleich mit Ziffern (14,3-28,7\%), die beim Uranhund kurz nach der Ischiadicusdurchschneidung ermittelt wurden, weitgehend kleiner. In 3 von 5 Versuchen (Versuch 1, 3 und 5) wurde das Maximum der Hämoglobinkonzentration in 30 Minuten nach Beginn der Staung, in übrigen 2 Versuchen nach 60 Minuten erreicht. Nach Beseitigung der Stauung wird 


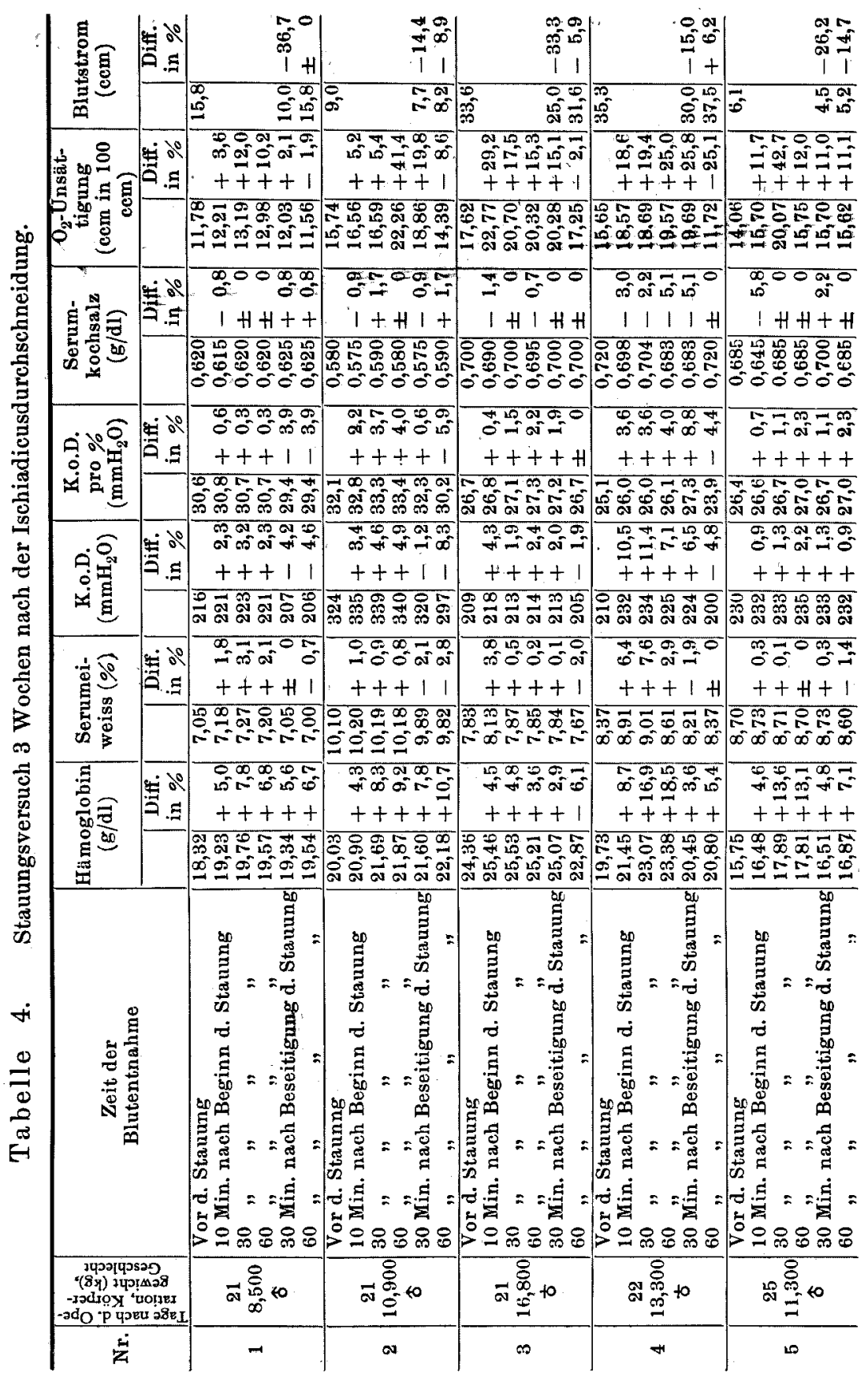


das Hämoglobin in 4 Versuchen (Versuch 1, 2, 4 und 5) vorübergehend herabgesetzt, bald darauf aber wieder erhöht, während in einem anderen Versuch (Versuch 3) es nach Behebung der Stauung allmählich abnimmt. Auch Serumeiweiss nimmt durch Stauung zu, die maximale Zunahme $(0 ; 3-7,6 \%)$ ist beinahe gleich der, die beim gesunden, unter denselben Bedingungen befindlichen Hund erhalten wird, aber im Vergleich mit der, die beim Uranhund nach dreistündigem Ablauf nach der Ischiadicusdurchschneidung ermittelt wurde, ziemlich geringer. Während indessen das Serumeiweiss im letzten Fall zu Beginn der Stauung nur vorübergehend zunimmt, später aber meistenfalls unter den Vorwert herabgesetzt wird, nimmt es beim dreiwöchigen Versuch während der Stauung unaufhaltsam zu, um erst nach Beseitigung der Stauung allmählich abzunehmen.

Zusammen mit der Zunahme des Serumeiweisses erhöht sich auch der k.o.D. Nur mit Ausnahme eines einzigen Falls (Versuch 4) ist die Erhöhung des k.o.D. gegenüber der, die beim gesunden Hund unter denselben Bedingungen gefunden wird, imallgemeinen geringer. Nach Beseitigung der Stauung fällt der k.o.D. parallel mit der Abnahme des Eiweisses ab. Da die prozentige Zunahme des k.o.D. gegenüber der Eiweisszunahme etwas grösșer ist, weist der Druck pro \% auch mehr oder minder Erhöhung, die aber im Vergleich mit der beim gesunden Hund unter denselben Bedingungen und auch gegenüber der bei Uranhund mit intaktem Ischiadicus im allgemeinen geringer ist. Serumkochsalz bietet keine wesentliche Veränderung dar.

Die $\mathrm{O}_{2}$-Unsättigung des venösen Blutes nimmt durch Stauung zu. Diese Zunahme unterschied sich nicht so sehr von der Zunahme, die beim Uranhund nach dreistündigem Ablauf nach der Ischiadicusdurchschneidung auftritt, aber beträchtlich kleiner im Vergleich mit der Zunahme beim gesunden Hund nach dreiwöchigem Ablauf nach der Ischiadicusdurchschneidung. Die Erklärung für die Frage, warum entnervtes Urangewebe, wie oben erwähnt,.im Vergleich zu entnervtem Normalgewebe während der Stauung weniger Sauerstoff aufzunehmen vermag, ist vorderhand schwer aufzustellen. Es verhält sich auch mit dem Kantharidingewebe (unten besprochen) ähnlich wie mit dem Urangewebe; das Gewebe nämlich, welches durch Uran oder Kantharidin geschädigt ist, dürfte unter der Bedingung eingeschränkter $\mathrm{O}_{2}$-Zufuhr wie der Stauung aus dem Blut nichthinreichend Sauerstoff aufzunehmen vermögen. Immeṛhin tritt die $\mathrm{O}_{2}$-Unsätttigung nach $\mathrm{Be}$ seitigung der Stauung allmählich an den Anfangswert heran:

Das Verhalten des Blutstroms nach Behebung der Stauung richtet 
sich beinahe ähnlich wie derselbe nach dreistündigem Ablauf nach der Nervendurchschneidung beim Uranhund. Mitunter gab es jedoch selbst denjenigen Fall (Versuch 4), in welchem der Blutstrom 60 Minuten nach Beseitigung der Stauung mehr als am Anfang beschleunigt war.

In der ersten Mitteilung ist bereits darauf hingewiesen worden, dass beim gesunden Hund schon einige Stunden nach der Durchschneidung des N. ischiadicus die Steigerung der Permeabilität der Kapillarwand für Wasser und Eiweiss nachzuweisen ist und ferner dass diese Permeabilitätssteigerung sich im Ablauf von einigen Wochen immer intensiver manifestiert. Die Feststellung, dass, wenn beim Hund, der nach dreiwöchigem Ablauf nach der Ischiadicusdurchschneidung mit Uran vergiftet worden war, der Stauungsversuch ausgeführt worden ist, die Zunahme der Hämoglobinkonzentration geringer als beim gesunden Hund unter gleichen Bedingungen und weitgehend geringer ist als beim Uranhund in einigen Stunden nach Ischiadicusdurchschneidung scheint zwar darauf hin zu deuten, dass im zuerst genannten Fall der Wasserdurchtritt durch die Kapillarwand weniger stark als in den letzteren zwei Fällen ist. Es ist indessen sehr strittig, ob man aus dieser Feststellung allein ohne weiteres darauf schliessen könnte, dass nach dreiwöchigem Ablauf nach Nervendurchschneidung die Kapillarpermeabilität im Vergleich mit derselben in den letzten zwei Fällen herabgesetzt sei. Denn im Hinblick auf die Tatsache, dass die Hämoglobinkonzentration schon vor Ausführung der Stauung beträchtlich erhöht gewesen ist, muss man den gleich unten anzuführenden Umständen Rechnung tragen, dass nämlich der Wassergehalt dieses degenerativ-atrophischen Urangewebes vermehrt ist, wobei obendrein in der betreffenden Gewebelücke die Wasseransammlung sich in erheblichem Masse findet, so dass auch bei Ausführung der Stauung Wasser nicht besonders in vermehrten Mengen vom Blute auf die Gewebeseite übertreten kann.

Was auch weiterhin die Veränderung des Serumeiweisses anbelangt, ist die Zunahme hier geringer als beim Normalhund unter gleicher Bedingung und auch geringer als beim Uranhund, der nach dreistündigem Ablauf nach derIschiadicusdurchschneidung dem Stauungsversuch unterworfen ist; der Aus- und Eintritt des Eiweisses erfolgt also nicht so lebhaft, wie in eben besprochenen Fällen. Die Veränderungen von k.o.D. und Druck pro \% gehen im allgemeinen parallel mit dem Serumeiweiss, ohne also merkliche Veränderungen aufzuweisen. 
Wenn man alle oben angeführte Daten in Erwägung zieht, so dürfte man wohl zu folgender Annahme berechtigt sein: Wenn man beim Hund nach dreiwöchigem Ablauf nach der Ischiadicusdurchschneidung, also zu einer Zeit, wo das zugehörige Gewebe degenerativatrophischen Veränderungen verfallen ist, die Uranvergiftung ausgelöst hat, so dürfte der Gasaustausch zwischen Blut und Gewebe, weil in diesem Stadium ohnehin schon durch den Einfluss der Nervenausschaltung am betreffenden Gewebe irgend wie kolloidale Veränderungen sich geltend gemacht haben müssen, selbst unter dem Einfluss der vönesen Stauung nicht so wesentliche Veränderungen erfahren, wie sie in der Norm zwangsläufig anzutreffen sind,-gleichviel, welches Verhalten auch die Permeabilität der zugehörigen Kapillarwand immer aufweisen möchte.

\section{Versuch an Kantharidinhund.}

In vorliegender Versuchsreihe wurden 8 Kantharidinhunde benutzt. Es wurde jedem einzelnen gesunden Hund eine 0,2\%ige Kantharidinlösung in Mengen von 0,05 ccm pro kg Körpergewicht subkutan injiziert; 15-35 Stunden später wurde mit dem Versuch begonnen.

\section{Staungsversuch an Kantharidinhund von der Ischiadicusdurchschneidung.}

Ca.15 Stunden nach Kantharidininjektion (Tabb. 5 u. 6) nahm Hämoglobin im Vergleich zur Norm um durchschnittlich $31,7 \% \mathrm{zu}$. Diese Zunahme kann auf die Bluteindickung bezogen werden, die dadurch entsteht, dass das infolge der Kapillarschädigung in Mitleidenschaft gezogene Gewebe stark quillt und Wasser aus dem Blut ansaugt. Die gegenüber dem Uranhund stärkere Zunahme des Hämoglobins zeigt an, dass Kantharidin auf das Gewebe stärker als Uran schädigend wirkt. Serumeiweiss nahm nur in geringem Masse ab. Die Herabsetzung der Eiweisskonzentration bei bestehender Bluteindickung spricht offensichtlich für kolossale Abnahme des Eiweisses; es ist dies wahrscheinlich darauf zurückzuführen, dass infolge von enormer Steigerung der Kapillarpermeabilität das Bluteiweiss mehr oder weniger samt dem Blutwasser ins Gewebe übergetreten ist.

K.o.D. war beträchtlich erniedrigt, und da die prozentuale Abnahme des k.o.D. grösser als die des Eiweisses war, fiel auch Druck pro \% erheblich ab. Dies dürfte wahrscheinlich auf den extravasalen 
Stoff- und Gasaustausch durch die Kapillaren. II.

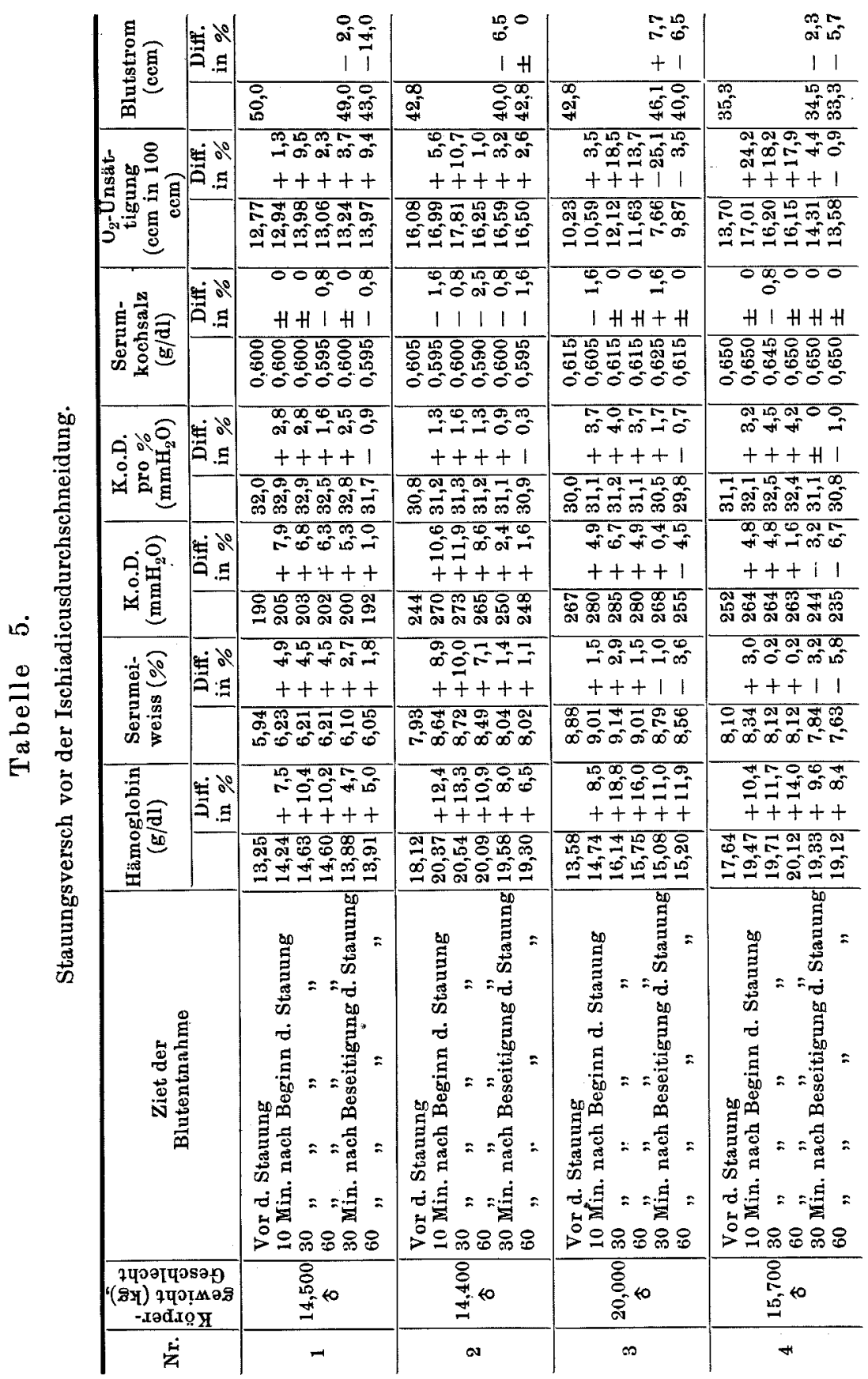


Tabelle

Durchschnittswerte vor und

\begin{tabular}{|c|c|c|c|c|c|c|c|c|c|}
\hline & \multicolumn{3}{|c|}{ Hämoglobin (g/dl) } & \multicolumn{3}{|c|}{ Serumeiweiss $(\%)$} & \multicolumn{3}{|c|}{ K.o.D. $\left(\mathrm{mmH}_{2} \mathrm{O}\right)$} \\
\hline & 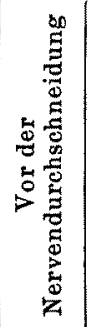 & 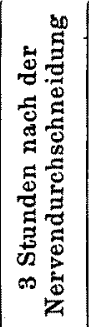 & 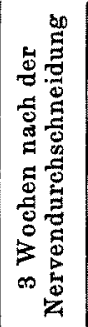 & 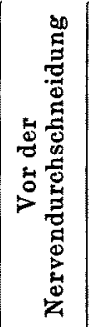 & 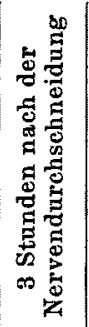 & 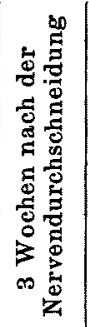 & 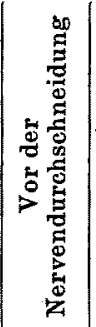 & 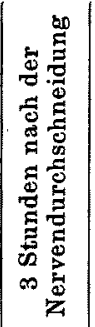 & 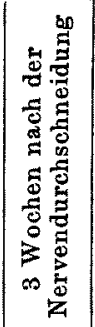 \\
\hline & \begin{tabular}{c|} 
Max. \\
18,12 \\
Min. \\
13,25
\end{tabular} & $\begin{array}{c}\text { Max. } \\
20,12 \\
\text { Min. } \\
13,30\end{array}$ & $\begin{array}{c}\text { Max. } \\
10,97 \\
\text { Min. } \\
7,99\end{array}$ & \begin{tabular}{|c|} 
Max. \\
8,88 \\
Min. \\
5,94
\end{tabular} & $\begin{array}{c}\text { Max. } \\
9,57 \\
\text { Min. } \\
6,55\end{array}$ & \begin{tabular}{|c|} 
Max. \\
7,50 \\
Min. \\
6,53
\end{tabular} & $\begin{array}{c}\text { Max. } \\
267 \\
\text { Min. } \\
190\end{array}$ & $\begin{array}{c}\operatorname{Max} . \\
298 \\
\operatorname{Min} . \\
210\end{array}$ & $\begin{array}{c}\text { Max. } \\
233 \\
\text { Min. } \\
200\end{array}$ \\
\hline Durchschnitt & 15,65 & 17,17 & 9,00 & 7,71 & 8,28 & 7,09 & 238 & 260 & \\
\hline Diff. in $\%$ & & $+9,7$ & $-42,5$ & & $+7,4$ & $-8,0$ & & $+9,2$ & $-8,8$ \\
\hline Norm & 11,88 & 12,57 & 13,28 & 7,70 & 8,35 & 8,41 & 256 & 313 & 295 \\
\hline $\left.\begin{array}{c}\text { Proz. Zu- u. Abnahme } \\
\text { gegenüber d. Norm }\end{array}\right\}$ & $+31,7$ & $+36,6$ & $-32,2$ & $+0,1$ & $-0,8$ & $-15,7$ & $-7,0$ & $-16,9$ & $-26,4$ \\
\hline
\end{tabular}

Übertritt der kleinmolekulären Eiweissteilchen bezogen werden. Aus hiesiger Klinik liegen die Angaben von Kimura u. Nakazawa, ${ }^{8}$ Saito u. Nakazawa $a^{9)}$ sowie Yasuda ${ }^{10)}$ vor, denen zufolge bei der Kantharidinvergiftung der k.o.D. und Druck pro \% erniedrigte Werte aufweisen. In Übereinstimmung mit dem Befund von Kashiwamura ${ }^{11)}$ nahm auch in vorliegendem Experiment der Serumkochsalz durch Kantharidinvergiftung zu.

Blutstrom und $\mathrm{O}_{2}$-Unsättigung des yenösen Blutes waren anscheinend nicht so bedeutsamen Schwankungen unterworfen.

Staungversuch: An oben erwähnten 5 Kantharidinhunden wurde der Stauungsversuch in üblicher Weise ausgeführt. Diese Ergebnisse sind in Tab. 6 angegeben.

Hämoglobin vermehrt sich durch Stauung. Die prozentuale $\mathrm{Zu}$ nahme beträgt im Maximum 18,8\%, im Minimum 10,4\%, war also gegenüber derselben beim gesunden Hund (2,1-7,8\%) höhergradig. In vorwiegender Mehrzahl der Fälle wird das Maximum in 30Minuten nach

11) Kashiwamura, Zikken Shokakibyo Gaku, 1935, 10, 1214. 
6.

nach der Nervendurchschneidung.

\begin{tabular}{|c|c|c|c|c|c|c|c|c|c|c|c|}
\hline \multicolumn{3}{|c|}{ K.o.D. pro $\%\left(\mathrm{mmH}_{2} \mathrm{O}\right)$} & \multicolumn{3}{|c|}{ Serumkochsalz (g/dl) } & \multicolumn{3}{|c|}{$\begin{array}{l}\mathrm{O}_{\mathrm{g}} \text {-Unsättigung } \\
(\mathrm{ccm} \text { in } 100 \mathrm{ccm})\end{array}$} & \multicolumn{3}{|c|}{ Blutstrom (ccm) } \\
\hline 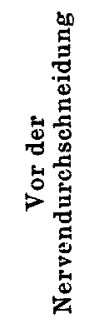 & 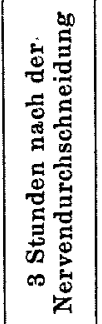 & 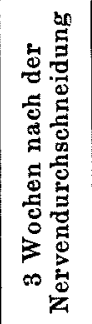 & 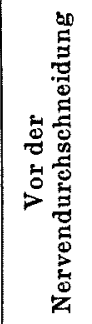 & 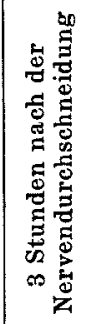 & 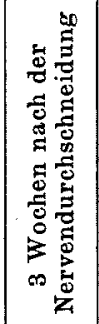 & 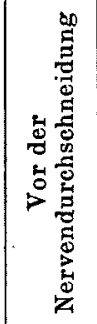 & 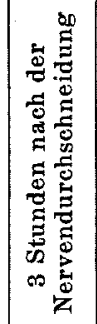 & 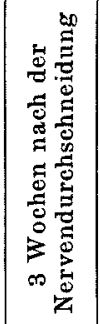 & 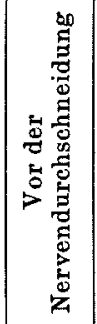 & 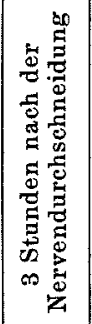 & 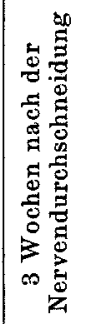 \\
\hline $\begin{array}{r}\operatorname{Max} . \\
\mathbf{3 2 , 0}\end{array}$ & $\mid \begin{array}{r}\operatorname{Max} \\
32,1\end{array}$ & $\begin{array}{r}\operatorname{Max} . \\
31,2\end{array}$ & $\begin{array}{c}\text { Max. } \\
0,650\end{array}$ & $\begin{array}{c}\text { Max. } \\
0,700\end{array}$ & \begin{tabular}{|c|} 
Max. \\
0,695
\end{tabular} & \begin{tabular}{|c|}
$\operatorname{Max}$. \\
16,08
\end{tabular} & \begin{tabular}{|}
$\operatorname{Max}$. \\
$\mathbf{9 , 9 0}$
\end{tabular} & $\underset{10,73}{M a x}$ & $\underset{50,0}{\operatorname{Max}}$ & $\begin{array}{r}\operatorname{Max} . \\
60,0\end{array}$ & $\begin{array}{r}\text { Max. } \\
60,0\end{array}$ \\
\hline $\begin{array}{l}\text { Min. } \\
\mathbf{3 0 , 0}\end{array}$ & \begin{tabular}{|} 
Min. \\
$\mathbf{3 1}, 0$
\end{tabular} \mid & $\underset{29,5}{\operatorname{Min} .}$ & $\underset{0,600}{\text { Min. }}$ & $\underset{\mathbf{0 , 6 2 0}}{\mathrm{Min} .}$ & $\begin{array}{c}\text { Min. } \\
0,620\end{array}$ & $\begin{array}{c}\text { Min. } \\
10,23\end{array}$ & \begin{tabular}{|} 
Min. \\
$\mathbf{3}, 89$
\end{tabular} & $\underset{1,89}{\operatorname{Min} .}$ & $\begin{array}{r}\text { Min. } \\
\mathbf{3 5 , 3}\end{array}$ & \begin{tabular}{|} 
Min. \\
22,2
\end{tabular} & $\underset{\mathbf{4 0 , 1}}{\text { Min. }}$ \\
\hline 31,0 & 31,4 & 30,6 & 0,617 & 0,650 & 0,664 & 13,19 & 7,74 & 4,65 & & & \\
\hline 33,5 & $\begin{array}{r}+\quad 1,3 \\
37,3\end{array}$ & $-1,3$ & 0,568 & $+5,3$ & $+7,6$ & 11,16 & $-41,3$ & $\begin{array}{r}-64,7 \\
10,60\end{array}$ & $\left\{\begin{array}{c}\operatorname{Max} . \\
60,0\end{array}\right.$ & $\begin{array}{c}\operatorname{Max} . \\
75,0 \\
\operatorname{Min}\end{array}$ & $\begin{array}{l}\operatorname{Max} . \\
50,0 \\
\operatorname{Min}\end{array}$ \\
\hline$-\quad 7,5$ & $-15,8$ & $-13,1$ & $+7,9$ & $+9,6$ & $+13,9$ & $+18,2$ & $-27,9$ & $-56,1$ & 20,0 & 30,0 & 34,6 \\
\hline
\end{tabular}

Beginn der Stauung erreicht. Nach Beseitigung der Stauung tritt eine Abnahme der Hämoglobinkonzentration ohne Ausnahme auf; diese Blutverdünnung tritt aber mehr verzögert ein als beim Uranhund.

Serumeiweiss erfährt durch Stauung eine Zunahme, das Maximum beträgt 2,9-10,0\%, ist stärker als das beim gesunden Hund (0,5-5,8\%), jedoch kleiner als die Zunahme des Hämoglobins. Durch Behebung der Stauung wird die Eiweisskonzentration ausnahmslos herabgesetzt. K.o.D. geht durch Stauung in die Höhe. Die maximale Erhöhung beträgt, von Fall zu Fall ziemlich verschieden, 4,8-11,9\%, also grösser als die beim gesunden Hund (5,1-9,0\%). Nach Beseitigung der Stauurg fällt der k.o.D., parallel mit dem Eiweiss gehend, ab. Da die prozentuale Zunahme des k.o.D., in Relation zur Eiweisszunahme gesetzt, nicht so gross wie die beim gesunden Hund ist, ist die Erhöhung von Druck pro \% (1,6-4,5\%) kleiner als im letzten Fall $(3,0-8,3 \%)$.

Serumkochsalz zeigt gegenüber der Norm keine bemerkbare Veränderung.

Die $\mathrm{O}_{2}$-Unsättigung des venösen Blutes nimmt zwar durch Stauung zu, der Grad der Zunahme ist aber niedriger als beim gesunden 
Hund. Nach Beseitigung der Stauung rückt sie allmählich an den Vorwert heran. Der venöse Blutsrom nimmt durch Beseitigung der Stauung im allgemeinen mehr oder minder ab.

Von oben angeführten. Befunden ist besonders interessant die Tatsache, dass das Hämoglobin durch Stauung auffallend stark zunimmt, während seine Abnahme (Blutverdünnung) relativ verzögert eintritt. Die Zunahme der Hämoglobinkonzentration deutet darauf hin, dass das Blutwasser in vermehrter Menge ins Gewebe übergetreten ist. Der verzögerte Eintritt der Blutverdünnung nach Beseitigung der Stauung lässt sich beim Uranhund vermissen. Yamagu $\mathrm{chi}^{5)}$ an hiesiger Klinik hat auch die Beobachtung gemacht, dass die Blutverdünnung nach Behebung der Stauung beim Kantharidinhund mehr verzögert eintritt als beim Uranhund und spricht die Vermutung dahin aus, dass dies wahrscheinlich darauf zurückzuführen sei, dass Kantharidin wohl in erster Linie die Kapillarschädigung hervorrufe, aber auch zugleich auf die Gewebszellen schädigend einwirke. Es ist also leicht aufzufassen, dass die Wiederherstellung von der an so stark geschädigtem Gewebe während der Stauung entstandenen Gewebsquellung, also die Entquellung nach Beseitigung der Stauung verzögert eintritt.

Weil die Zunahme des Eiweisses zwar grösser als die beim gesunden Hund ist, aber die Erhöhung des k. o.D. keine merkliche Differenz aufweist, fällt Druck pro \% ausserordentlich ab, was darauf hinweist dass kleinmolekuläre Eiweissteilchen wegen gesteigerter Kapillarpermeabilität ins Gewebe übergetreten ist.

Die geringfügige Erhöhung der $\mathrm{O}_{2}$-Unsättigung venösen Blutes durch Stauung dürfte wohl dadurch bedingt sein, dass durch Kantharidinvergiftung die Gewebe- und Kapillarschädigung sich einstellen und dadurch der Gasaustausch zwischen Blut und Gewebe gestört ist. Wallace u. Pellini $\mathbf{i}^{12}$ sind der Meinung, dass die durch sog. Nierengifte (Uran und Kantharidin) hervorgerufene Azidosis die Kapillarschädigung hervorruft und dadurch die Sauerstoffzufuhr von angemessenen Mengen zum Gewebe behindert. Auch Kimura ${ }^{6}$ hat nachgewiesen, dass bei Kantharidinvergiftung unter der Gewebsschädigung der Gasaustausch zwischen Blut und Gewebe gestört ist und auch die Atmungsfähigkeit der roten Blutkörperchen selbst herabgesetzt ist. Beim Uranhund hingegen ist die Erhöhung der venösen $\mathrm{O}_{2}$-Unsättigung durch Stauung annähernd gleichgradig wie in der Norm. Kimura $a^{6)}$ hat bereits darauf aufmerksam gemacht, dass der Gasumsatz

12) Walla ce u. Pellini, Proc. Soc. Exp. Biol. a. Med., 1920-1921, 18, 115. 
der Gewebe beim Uranhund von demselben beim gesunden Hund nicht so abweicht.

Vergleicht man die Permeabilität der Kapillaren für Wasser und Eiweiss beim Kantharidin- und Uranhund gegeneinander, so erkennt man, dass sie beim Uranhund stärker als heim Kantharidinhund ist, weil beim Uranhund durch Stauung die Hämoglobinkonzentration beträchtlich und zwar in stärkerem Masse als beim Kantharidinhund zunimmt und zumal die Eiweisskonzentration im allgemeinen unter den Vorwert herabfällt, während dies beim Kantharidinhund nicht der Fall ist.

\section{Staungsversuch nach dreistündigem Ablauf} nach der Ischiadicusdurchschneidung.

4 Kantharidinhunden wurde 15-18 Stunden nach dem Stauungsversuch, der an einem Schenkel durchgeführt wurde, also ca. 35 Stunden nach der Kantharidininjektion N. ischiadicus des anderen Schenkels durchschnitten und 3 Stunden später analogener Stauungsversuch angestellt.

Beim Kantharidinhund mit durchschnittenem $N$.ischiadicus nimmt das Hämoglobin venösen Blutes, wie Tab. 6 zeigt, im Vergleich mit demselben beim normalen, denervierten Hund in Durchschnitt um $36,6 \% \cdot z u$, während das Eiweiss hierbei keinen grossen Unterschied aufweist. K.o.D. und Druck pro \% erfahren hierbei erhebliche Erniedrigungen. Es lassen sich sonst kaum nennenswerte Veränderungen erkennen, abgesehen von der Abnahme der $\mathrm{O}_{2}$-Unsättigung venösen Blutes.

Ein Vergleich mit den Zifferwerten, die vor der Nervendurchschneidung ermittelt wurden, ergibt, dass nach der Nervendurchschneidung sich durchschnittlich das Hämoglobin um 9,7\% und das Serumeiweiss um 7,4\% vermehrt, während der Druck pro \% in annähernd gleicher Höhe bleibt. Hieraus kann erschlossen werden, dass durch Ischiadicusdurchschneidung die Permeabilität der Kapillaren für Blutwasser erhöht ist. Es ist bereits oben angeführt worden, dass beim Uranhund, da hier die Kapillarpermeabilität durch die Uranwirkung schon auf abnorme Lage eingestellt, d. h. enorm gesteigert ist, das Blut selbst durch Ischiadicusdurchschneidung fast keine weitere Veränderung erfährt. Hieraus geht klar hervor, dass das Uran die Kapillaren in stärkerem Masse als das Kantharidin zu schädigen vermag.

$\mathrm{O}_{2}$-Unsättigung venösen Blutes nimmt durch Nervendurchschnei- 
dung ab, was auf dem Zuwachs des Blutstroms beruht.

Staungsversuch: Die Ergebnisse sind in Tab. 7 wiedergegeben.

Hämoglobin nimmt durch Stauung in allen Fällen zu. Die Zunahme beträgt 9,6-19,5\%, ist also ziemlich grösser als die beim gesunden Hund unter denselben Bedingungen gefundene Zunahme (3,616,8\%). Auch Serumeiweiss nimmt durch Stauung zu, die maximale Vermehrung $(0,7-5,3 \%)$ ist etwas kleiner als die Zunahme beim Normalhund (2,5-8,1\%). Durch Behebung der Stauung erfährt die Hämoglobin- und Eiweisskonzentration Abnahme. Das Verhalten der Veränderung des k. o.D. richtet sich nach demselben des Eiweisses. Durch Stauung erhöht sich nämlich der k.o.D., durch Behebung derselben sinkt er ab. Weil die Erhöhung des k.o.D. im Vergleich zur Eiweisszunahme ziemlich grösser ist, steigt Druck pro \% um ein Geringes an. In bezug auf die Veränderung des Serumkochsalzes besteht kein bemerkbarer Unterschied gegenüber dem Normalhund. Die venöse $\mathrm{O}_{2}$ Unsättigung wächst durch Stauung zu, dieser Zuwachs ist aber geringer als der beim Normalhund. Nach Beseitigung der Stauung rückt sie graduell an den Anfangswert heran. Der venöse Blutstrom setzt sich durch Beseitigung der Stauung ausnahmslos unter den Vorwert herab, um sich dann aber dem Vorwert zu nähern. Derartiger Befund ist ähnlich dem Befund, der beim Normal-und Uranhund nach dreistündigem Ablauf nach der Durchschneidung des $\mathrm{N}$. ischiadicus erhoben wird.

Aus der Feststellung, dass die Zunahme derHämoglobinkonzentration beim Kantharidinhund sowohl ge'genüber derselben vor der Nervendurchschneidung wie auch im Vergleich mit der Zunahme, die beim Normalhund nach der Ischiadicusdurchschneidung gefunden wurde, grösser ist, kann darauf geschlossen werden, dass beimKantharidinhund nach der Ischiadicusdurchschneidung die Permeabilität der Kapillaren für Wasser offensichtlich gesteigert ist. Der Grad dieser Steigerung ist jedoch geringer als beim Uranhund, woraus zu schliessen ist, dass bei der Uranvergiftung die Kapillarpermeabilität in stärkerem Masse als bei der Kantharidinvergiftung gesteigert ist. Die Zunahme des Serumeiweisses durch Stauung ist im Verhältnis zur Hämoglobinzunahme im allgemeinen sehr geringfügig. Es ist dies ein Beweis dafür, dass das Bluteiweiss unter dem Einfluss der Stauung durch die Kapillarwand hindurch ins Gewebe abfiltriert wird. Dieser extravasale Übertritt des Eiweisses erfolgt in stärkerem Masse sowohl als beim Kantharidinhund vor der Nervendurchschneidung wie auch als 
Stoff- und Gasaustausch durch die Kapillaren. II.

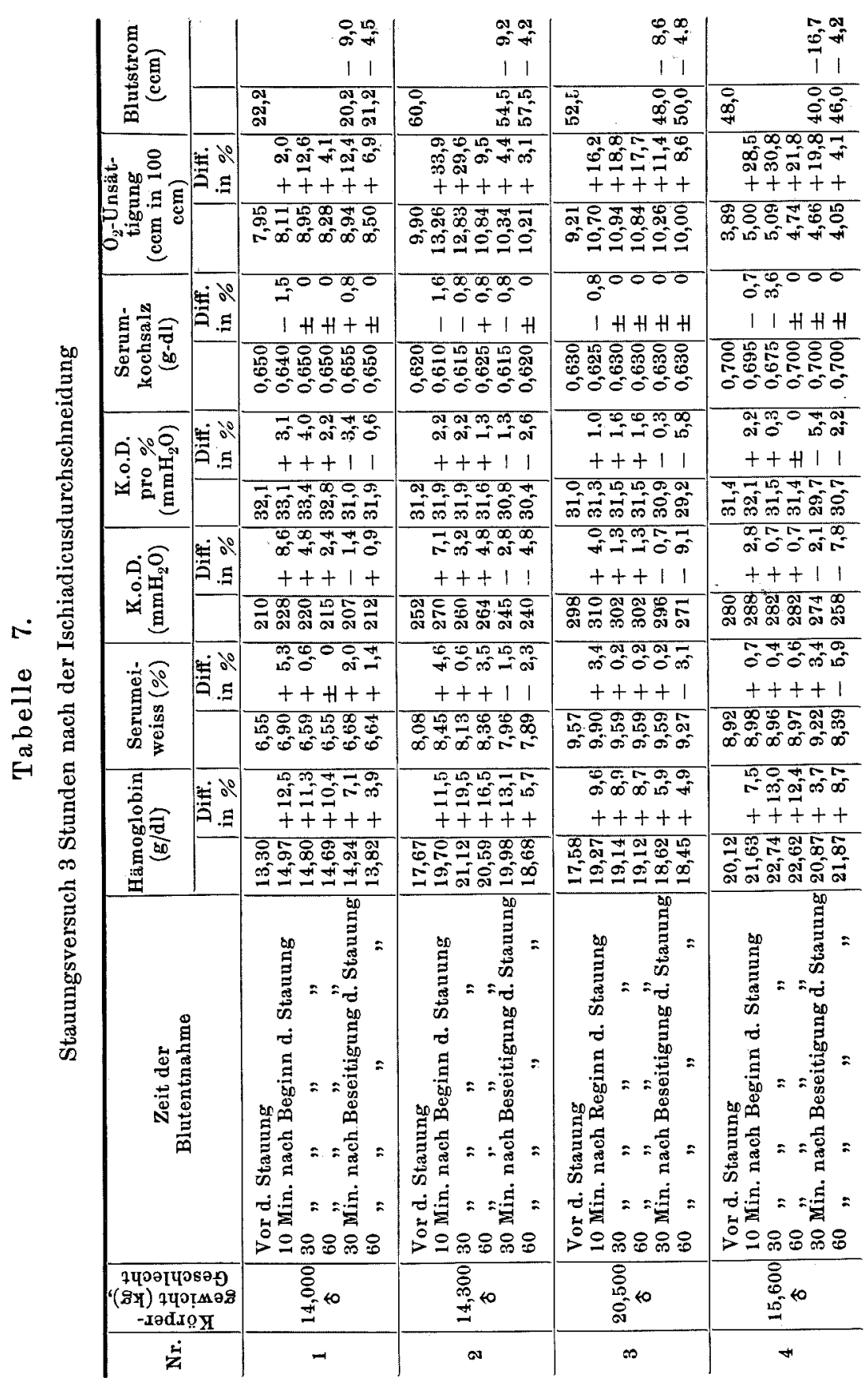


beim Normalhund mit durchschnittenem Ischiadicus. Beim Uranhund mit durchschnittenem Ischiadicus fällt das Eiweiss am Ende der Stauung unter den Wert vor der Stauung ab, was einen lebhaften Übertritt des Eiweisses durch die Kapillarwand hindurch ins Gewebe anzeigt. Bei der Kantharidinvergiftung wird ein derartiger Befund vermisst. Hieraus ist anzunehmen, dass die Permeabilität der dem Einfluss der Stauung unterliegenden Gefässe für Eiweiss weniger ausgeprägt ist als bei Uranvergiftung.

Im Übrigen der Umstand, dass, weil die Erhöhung des k.o.D. durch Stauung grösser als die Eiweisszunahme ist, dementsprechend auch Druck pro \% mehr oder weniger erhöhte Werte aufweist, spricht dafür, dass unter derartiger Bedingung Molekularaggregate des Bluteiweisses nach kleindisperser Seite hin verschoben sind.

\section{Staungsversuch nach dreiwöchigem Ablauf nach der Ischiadicusdurchschneidung.}

Jedem einzelnen Versuchstier wurde der N. ischiadicus auf einer Seite durchschnitten und nach dreiwöchigem Ablauf, wo an zugehörigen Kapillaren und Geweben ausgesprochene degenerative Veränderungen auftraten, das Versuchstier mit Kantharidin vergiftet und ca. 35 Stunden später an der Extremität mit durchschnittenem Ischiadicus der Stauungsversuch ausgeführt.

An 4 Hunden gewonnene Versuchsergebnisse sind in Tab. $8 \mathrm{zu}-$ sammengestellt.

Nach dreiwöchigem Ablauf nach der Ischiadicusdurchschneidung erfahren Hämoglobin, Serumeiweiss und $\mathrm{O}_{2}$-Unsättigung des venösen Blutes, wie aus in Tab. 4 aufgezeichneten Ziffern ersichtlich ist, auffallende Abnahmen. Parallel mit Abnahme der Eiweisskonzentration sinken auch der k.o.D. sowie Druck pro $\%$ erheblich ab. Serumkochsalz nimmt um ein Geringes zu. Blutstrom in der Femoralvene zeigt in eben ähnlicher Weise wie in direktem Anschluss an die Nervendurchschneidung die Tendenz zu mehr oder weniger Beschleunigung. $\mathrm{O}_{2}$-Unsättigung venösen Blutes nimmt deutlich ab.

Was die Veränderungen der Blutkonzentration anbelangt, treten bemerkenswerte Befunde in Erscheinung. Während nämlich beim Uranhund in direktem Anschluss an die Nervendurchschneidung keine wesentliche Veränderung im Hämoglobingehalt also in der Blutkonzentration auftritt, besteht beim Kantharidinhund eine augenfällige Bluteindickung. Allein nach 3 Wochen, wo sich am Gewebe degene- 
Stoff- und Gasaustauseh durch die Kapillaren. II.

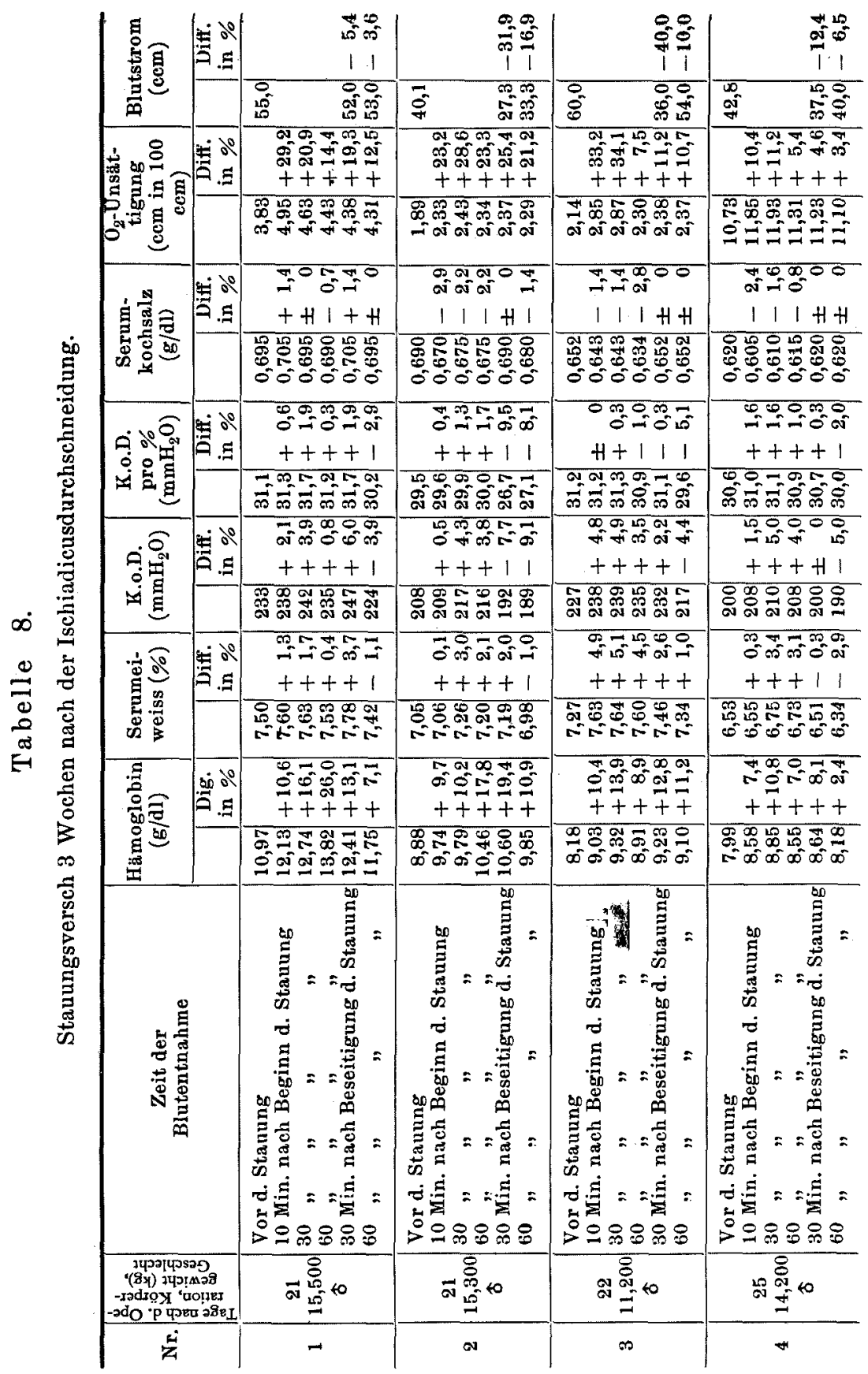


rative Veränderungen geltend machen, kommt è bei Uranvergiftung zur Bluteindickung, wohingegen bei Kantharidinvergiftung eine Blutverdünnung in den Vordergrund tritt. Da die Blutkonzentration vor der Nervenduchschneidung, nicht ermittelt wurde, kann ich leider kaum entscheidend angeben, dennoch soviel vermuten, dass es sich hierbei nicht um den Auftritt der Anämie nach der Nervendurchschneidung handelt, weil beim Normal- und Uranhund unter gleichen Bedingungen eine Bluteindickung zum Vorschein kommt. Nach Kimura u.a. ist die Gewebsschädigung nach Kantharidin höhergradig als nach Uran. Mit dieser Beziehung allein lässt sich dennoch eine derart hochgradige Blutverdünnung, wie sie oben erwähnt worden ist, kaum erklären.

Nach alledem ist die Erklärung für jene hochgradige Blutverdünnung, welche dann eintritt, wenn degenerative Gewebe durch Kantharidin geschädigt worden sind, recht schwer aufzustellen.

Es sei nun an Hand der Tab. 8 über die Ergebnisse des Stauungsversuchs betrachtet.

Hämoglobin nimmt durch Stauung zu. Diese Zunahme (10,8$26,0 \%)$ ist ein wenig grösser als beim gesunden Hund unter denselben Bedingungen $(9,2-22,0 \%)$ und auch etwas grösser als beim Kantharidinhund in kurz der Ischiadicusdurchschneidung nachfolgender Zeit (9,2-19,5\%). Nach Beseitigung der Stauung nimmt das Hämoglobin in allen Fällen ab. Auch Serumeiweiss wird durch Stauung vermehrt gefunden. Die maximale Vermehrung beträgt 1,7-5,1\%, unterscheidet sich also nicht so sehr von Werten in eben besprochenen zweierlei Fällen. Nach Behebung der Stauung sinkt das Serumeiweiss ab. Entsprechend der Zunahme des Serumeiweisses erhöht sich auch der k.o.D.. Diese Erhöhung ist jedoch geringer als beim gesunden Hund unter gleicher Bedingung. Durch Behebung der Stauung sinkt der k. o. D. parallel mit der Abnahme des Eiweisses ab. Abgesehen von einem Ausnahmefall (Versuch 4) ist die prozentuale Zunahme des k.o.D. einigermassen grösser als die Zunahme des Eiweisses, so dass sich Druck pro \% erhöht.

Serumkochsalz bietet keine wesentliche Veränderung dar. $\mathrm{O}_{2}-\mathrm{Un}$ sättigung venösen Blutes nimmt durch Stauung zu. Die prozentuale Zunahme unterscheidet sich kaum von derselben beim Kantharidinhund im direkten Anschluss an die Ischiadicusdurchschneidung, ist aber deutlich geringer als beim gesunden Hund nach dreiwöchigem Ablauf nach der Ischiadicusdurchschneidung. Nach Beseitigung der Stauung tritt die $\mathrm{O}_{2}$-Unsättigung allmählich an das frühere Niveau 
heran. Das Verhalten des Blutstromes nach Beseitigung der Stauung ist beinahe ähnlich demselben beim Kantharidintier kurz nach der Nervendurchschneidung.

Von den oben erwähnten Ergebnissen des Stauungsversuchs ist hervorzuheben, dass die Zunahme der Hämoglobinkonzentration recht erheblich ist, was offenbar ein Beweis dafür ist, dass beim degenerativen Schenkel, der unter dem Einfluss derStauung steht, der Durchtritt des Wassers durch die Kapillarwand ausserordentlich leicht vor sich geht. Aus dem Umstand, dass dabei die Veränderung des Serumeiweisses gegenüber anderen Fällen etwas geringergradig oder davon nicht so verschieden ist, kann darauf geschlossen werden, dass die Differenz zwischen der Zunahme des Hämoglobins und Eiweisses mehr oder weniger grösser ist als bei anderen Fällen, was darauf hinweist, dass die Permeabilität der Kapillarwand für Eiweiss intensiv ist. Der Grund dafür, dass die Erhöhungen von k. o. D. und Druck pro \% kleiner als beim gesunden Hund unter im übrigen gleichen Bedingungen sind, wäre darin zu suchen, dass infolge von gesteigerter Permeabilität der Kapillaren für Bluteiweiss kleinmolekuläre Eiweissteilchen durch den Einfluss der Stauung mit Leichtigkeit durch sie durchtreten können. Dass die $\mathrm{O}_{2}$-Unsättigung venösen Femoralblutes sowohl im direkten Anschluss an die Ischiadicusdurchschneidung wie auch nach einigen Wochen durch Stauung in geringerem Masse als beim gesunden Tier zunimmt, dürfte wahrscheinlich so aufgefasst werden, dass der $\mathrm{O}_{2}-\mathrm{Be}$ darf bzw. die Atmungsfunktion des unter dem Einfluss der Stauung stehenden Kantharidingewebes gegenüber dem intakten Gewebe unter gleicher Bedingung mehr herabgesetzt ist.

\section{Zusammenfassung.}

1. Beim Uran- und Kantharidinhund ist die Durchlässigkeit der Kapillaren für Wasser und Eiweiss gesteigert. Es kommt hierbei durch physiko-chemische Veränderungen des betreffenden Gewebes zur Bluteindickung. Diese Bluteindickung tritt beim Kantharidintier mehr manifest zutage, dies deutet offensichtlich darauf hin, dass die schädigende Wirkung auf das Gewebe mehr dem Kantharidin als dem Uran zukommt. Bei der Wirkung des ersteren vollzieht sich der extravasale Austritt des Eiweisses besonders ausgeprägt. Bei der Einwirkung der beiden Gifte ist das Bluteiweiss nach grossdisperser. Seite hin verschoben.

2. Wenn die Femoralvene des Urantiers abgeklemmt ist, so nimmt 
die Hämoglobinkonzentration des zugehörigen venösen Blutes in stärkerem Masse als beim gesunden Hund zu, während die Eiweisskenzentration hingegen hierbei eher abnimmt, was für eine stärkere Steigerung der Kapillarpermeabilität durch Uran spricht. Der kolloidosmotischeDruck pro \% Eiweiss des Bluteserfährt beimUranhund durch Stauung durchaus nicht só deutliche Erhöhung, wie dies beim gesunden Hund der Fall ist. Auch beim Kantharidintier ist die Eindickung venösen Blutes durch Stauung wwar stärker als beim Normaltier, aber schwächer als beim Urantier, indessen tritt die Wiederherstellung davon nach Behebung der Stauung gegenüber dem Urantier ausserordentlich verzögert ein, was auf stärkere Gewebsschädigung hinweist. Beim Kantharidintier nimmt das Serumeiweiss, im Gegensatz zum Urantier, im Laufe der Staung zu. Es ist dies darauf zurückzuführen, dass bei der Kantharidinwirkung die Schädigung der Kapillaren geringergradig als bei der Uranwirkung ist, so dass die Durchlässigkeit für Eiweiss nicht so sehr wie bei der Uranwirkung gesteigert ist.

3. Nach Uran ist der $\mathrm{O}_{2}$-Defizit, also die $\mathrm{O}_{2}$-Unsättigung venösen Blutes während der Stauung grösser als beim Normaltier. Während diese $\mathrm{O}_{2}$-Unsättigung beim Normalen durch Behebung der Stauung eher unter den Wert vor Provokation derselben herabgesetzt ist, wird beim Uranhund eine derartige Herabsetzung meistens vermisst. Auch der Blutstrom erfährt nach Beseitigung der Stauung niemals eine Erhöhung wie beim Normalen, sondern im allgemeinen eher eine Herabsetzung.

4. Beim Urantiere nach Ablauf von mehreren Stunden nach der Ischiadicusdurchschneidung treten am Blut aus der Femoralvene Veränderungen des Hämoglobins und Eiweisses noch nicht so bemerkbar in den Vordergund; nach Ablauf von einigen Wochen, wo am zuggehörigen Gewebe degenerative Veränderungen schon sich geltend machen, kommt es zu erheblicher Zunahme der Hämoglobinkonzentration, also zu auffallender Bluteindickung, was auf einen lebhaften Austritt des Blutwassers durch die Kapillaren hinweist. A Aus dem Ausbleiben der Zunahme des Eiweissgehaltes kann man auf den intensiven extravasalen Austritt des Eiweisses schliessen. Kolloid-osmotischer Druck und Druck pro \% Eiweiss sinken schon von kurz der Nervendurchschneidung nachfolgender Zeit an, im Gegensatz zum gesunden Tier, ab und nach Ablauf von mehren Wochen in immer stärkerem Masse. Hieraus dürfte erschlossen werden, dass die Nervenduchschneidung wahrscheinlich zum extravasalen Übertritt kleinmolekulärer Eiweissteilchen führt.

Beim Kantharidintier führt die Nervendurchschneidung zu star- 
ker Eindickung des venösen Blutes, daraus kann man ersehen, dass die Durchlässigkeit der Blutkapillaren für Wasser durch diesen Eingriff gesteigert ist. Darin besteht allerdings ein scharf begrenzter Unterschied vom Urantier; beim Urantier nämlich hat die Kapillarschädigung bereits vor diesem Eingriff in stärkerem Masse als beim Kantharidintier bestanden, so dass in direktem Anschluss an die Nervendurchschneidung eine weitere Bluteindickung nicht vorkommen kann. Nach einigen Wochen tritt eine Abnahme des Hämoglobins als Ausdruck der Blutverdünnung auf, dadurch unterscheidet sich das Kantharidintier vom Urantier. Kolloid-osmotischer Druck und Druck pro \% Eiweiss schlagen nach der Nervendurchschneidung geradeswegs den Weg zum Absinken ein.

5. Beim Normalhund vollzieht sich durch Nervendurchschneidung neben dem Zuwachs venösen Blutstromes auch eine erhebliche Verminderung der $\mathrm{O}_{2}$-Defizit des venösen Blutes. Dieser Befund klingt aber einige Wochen nach der Nervendurchschneidung nach und nach ab. Beim Uranhund hat die Nervenduchschneidung durchaus nicht wesentliche Veränderungen des Blutstroms und der $\mathrm{O}_{2}$-Unsättigung venösen Blutes zur Folge, sondern beide Grössen haben vielmehr mit dem Zeitverlauf die Tendenz zur Zunahme. Das ist dahin zu erklären, dass durch Uranwirkung an zugehörigen Gefässen stärke Schädigungen sich eingestellt haben und zwar derart, dass es durch Nervendurchschneidung nicht zu nachweisbarer Gefässdilatation kommt, wie dies in der Norm der Fall ist. Beim Kantharidintier tritt durch Nervendurchschneidung in ebenso ähnlicher Weise, wie in der Norm die Gefässerweiterung mit verstärkter Beschleunigung des Blutstomes und Abnahme der $\mathrm{O}_{2}$-Unsättigung venösen Blutes in Erscheinung.

6. Wenn man beim Uranhund mit durchschnittenem Ischiadicus durch Abklemmen der Femoralvene die Stauung provoziert, so erfolgt der extravasale Wasseraustritt intensiver als in der Norm, da die Kapil-. laren ausgesprochen durchlässig sind und ferner die Gewebe durch Stauung stark aufquellen; die Wiederherstellung des vermehrten Hämoglobins im venösen Blut zum früheren Niveau nach Beseitigung der Stauung ist ganz verzögert. Hingegen nimmt das Bluteiweiss, im Gegensatz zum gesunden Hund, nach eine Stunde dauernder Stauung eher ab und zwar in den meisten Fällen unter die Konzentration vor der Stauung. Daran erkennt man, dass die durch Uran geschädigten Kapillaren unter dem Einfluss der Stauung überaus leicht das Eiweiss durchlassen. Derartige Verminderung des Eiweisses besteht nach Beseitigung der Stauung noch weiter fort. 
Beim Uranhund nach dreiwöchigem Ablauf nach derNervendurch schneidung ist die Zunahme des Hämoglobins venösen Blutes durcl Stauung weniger gross als in der Norm. Da aber die Hämoglobin konzentration sich hierbei bereits vor Provokation der Stauung erheb. lich gesteigert hat, hat man keinen Grund, aus schwächerer Zunahme der Hämoglobinkonzentration ohne weiters darauf zu schliessen, dask die Durchlässigkeit der Kapillaren für Wasser nach Ablauf von meh. reren Wochen nach Nervendurchschneidung herabgesetzt wäre. Diє Veränderungen von Bluteiweiss und kolloid-osmotischem Druck durch Stauung sind äusserst geringfügig, das deutet ohne Zweifel darauf hin, dass der Stoffaustausch zwischen Blut und Gewebe infolge der im Gewebe entstandenen kolloidalen Veränderungen und dergleichen selbst auf den Eingriff wie die Staung nicht mehr so ansprechbar wie in der Norm ist. Kolloid-osmotischer Druck venösen Blutes sowie Druck pro $\%$ erhöhen sich während der Stauung.

Beim Kantharidinhund erfolgt die Zunahme der Hämoglobinkonzentration durch Stauung weniger stark als beim Uranhund, auch hier bleibt jene während der Stauung auftretende Abnahme der Eiweisskonzentration, welche beim Urantier unfehlbar anzutreffen ist, aus, was auch darauf hinweist, dass das Kantharidin die Durchlässigkeit der Kapillaren weniger stark als das Uran zu steigern vermag. Auch beim Kantharidintier erhöhen sich der kolloid-osmotischeDruck des venösen Blutes sowie Druck pro \% durch Stauung mehr oder minder.

7. Bei den Vergiftungen, dem Uran- und Kantharidinhund, ist gemeinsam, dass die $\mathrm{O}_{2}$-Unsättigung des venöşen Blutes sowohl im direkten Anschluss an die Nervendurchschneidung als auch nach mehrwöchigem Ablauf durch Stauung geringere Zunahme als beim gesunden Hund erfährt. Der $\mathrm{O}_{2}$-Bedarf bzw. die Atmungsfunktion des unter dem Einfluss der Stauung stehenden Gewebes dürfte also bei beiden Vergiftungen mehr beeinträchtigt sein als beim Gesunden. 